\title{
A Rare Presentation of Isolated Right Colon Ischemia: The Mass-Forming Variant
}

\author{
M'hamed Turki $^{1}$, Anumita Chakraborti ${ }^{2}$, Saif Bella ${ }^{3}$, Amine Hila ${ }^{3}$, Ali Timsar ${ }^{4}$
}

1. Internal Medicine, United Health Services, Johnson City, USA 2. Gastroenterology and Hepatology, State University of New York (SUNY) Upstate Medical University, Syracuse, USA 3. Gastroenterology and Hepatology, United Health Services, Johnson City, USA 4. Pathology, United Health Services, Johnson City, USA

Corresponding author: Ali Timsar, ali.timsar@nyuhs.org

\begin{abstract}
Ischemic colitis (IC), the most common gastrointestinal ischemia, remains an enigmatic disease with a wide array of pathogenic mechanisms and injuries along with variable outcomes. Among this group, isolated right colon ischemia (IRCI) appears to be a distinct entity, with its own pathophysiology, clinical presentation, and higher morbidity and mortality compared to left-sided colitis. IRCI is the most common site of massforming ischemic colitis. Colonoscopy with biopsy remains the key to diagnosis for this former entity. IRCI management is the same as for other IC and complete resolution of the mass is expected within weeks.
\end{abstract}

Review began 01/07/2021 Review ended 01/26/2021 Published 01/31/2021

\section{(c) Copyright 2021}

Turki et al. This is an open access article distributed under the terms of the Creative Commons Attribution License CC-BY 4.0., which permits unrestricted use, distribution, and reproduction in any medium, provided the original author and source are credited.
Categories: Pathology, Radiology, Gastroenterology

Keywords: ischemic colitis, mass-like lesions in colon, right sided colon ischemia

\section{Introduction}

Ischemic colitis (IC), the most common gastrointestinal ischemia, remains an enigmatic disease with a wide array of pathogenic mechanisms and variable outcomes [1]. Compared to left-sided colitis, isolated right colon ischemia (IRCI) has higher morbidity and mortality, it is also the most common site of mass-forming ischemic colitis [2,3]. Here, we report two cases of IRCI that present as a pseudotumor and emphasize the singularity and implications of this variant.

\section{Case Presentation}

\section{Case 1}

An 82-year-old Caucasian female with a history of hypertension, gastroesophageal reflux disease, dyslipidemia and gout, presented with right lower quadrant (RLQ) abdominal pain followed by a loose nonbloody bowel movement. Her symptoms were associated with tiredness, nausea, subjective fever and chills. She was hemodynamically stable on presentation. Physical examination revealed RLQ fullness to palpation. Initial blood work was remarkable for white blood cell count (WBC) $20.9 \times 10^{9} / \mathrm{mm}^{3}$, hemoglobin $11.8 \mathrm{~g} / \mathrm{dl}$ and blood urea nitrogen (BUN) $25 \mathrm{mg} / \mathrm{dl}$. Computed tomography (CT) of the abdomen and pelvis showed a $6.3 \mathrm{~cm}$ mass-like thickening of the proximal right colon near the ileocecal valve which was concerning for colonic neoplasm (Figure 1).

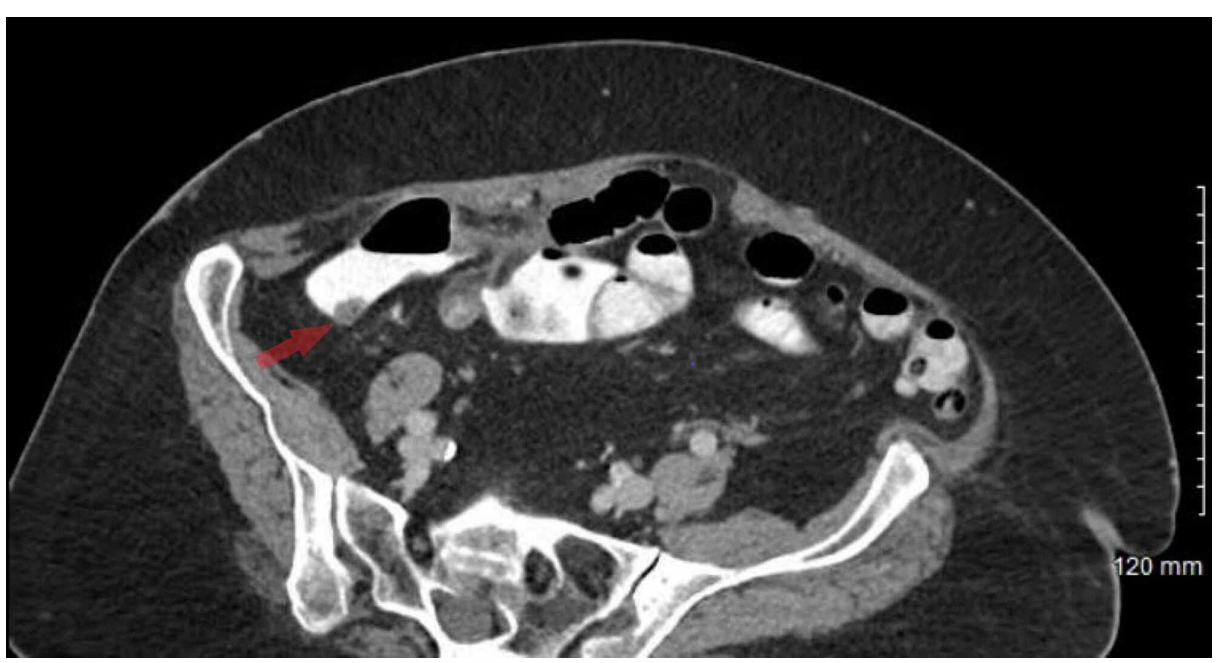

FIGURE 1: Axial view of a CT of the abdomen and pelvis with oral and intravenous contrast 


\section{Cureus}

Mass-like thickening of the proximal right colon near the ileocaecal valve, measuring $6.3 \mathrm{~cm}$ in length, involving the proximal aspect of the ascending colon and the adjacent cecum with adjacent fat stranding (Transparent red arrow).

Carcinoembryonic antigen was within normal limits. Stool studies were negative for enteric pathogens or Clostridium difficile infection and fecal calprotectin level was elevated at $309 \mathrm{mcg} / \mathrm{g}$. Colonoscopy demonstrated an area of moderately congested and ulcerated mucosa in the ascending colon and cecum suspicious for ischemic colitis (Figure 2).

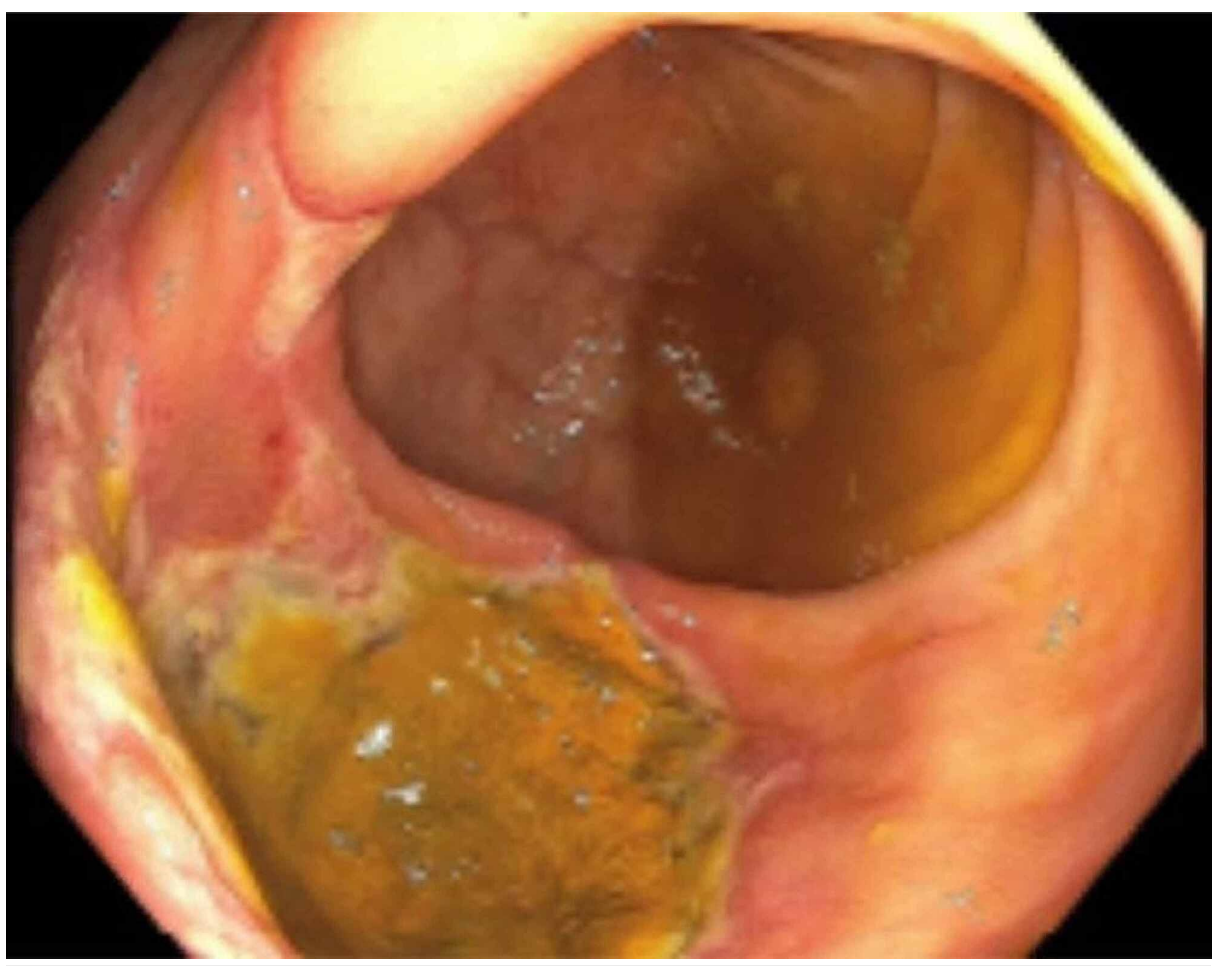

\section{FIGURE 2: Colonoscopy image, at the level of the cecum}

A sample of the multiple $20 \mathrm{~mm}$ ulcers found in the cecum and ascending colon.

Biopsies showed ulceration and granulation tissue and ruled out dysplasia or malignancy. The patient was treated successfully with intravenous (IV) fluids, bowel rest regimen and IV antibiotics.

\section{Case 2}

A 70-year-old male with a pertinent history of coronary artery disease (CAD), hypertension, prediabetes and hypothyroidism underwent an off-pump coronary artery bypass graft. Ten days later, the patient presented to the hospital complaining of sharp colicky RLQ abdominal pain and dark stools. Blood pressure was down to $80 / 40$ and improved with intravenous fluid resuscitation. Physical examination revealed RLQ tenderness. Blood workup was remarkable for sodium $134 \mathrm{mmol} / \mathrm{L}$, BUN $25 \mathrm{mg} / \mathrm{dl}$, lactate dehydrogenase (LDH) 673 U/L, WBC $18.9 \times 10^{9} / \mathrm{L}$ and hemoglobin $9 \mathrm{~g} / \mathrm{dl}$. CT angiography of the abdomen and pelvis disclosed a 6-cm cecal mass concerning for colon cancer, high-grade stenosis of the proximal celiac artery and mild stenosis of the proximal mesenteric artery (Figure 3). 


\section{Cureus}

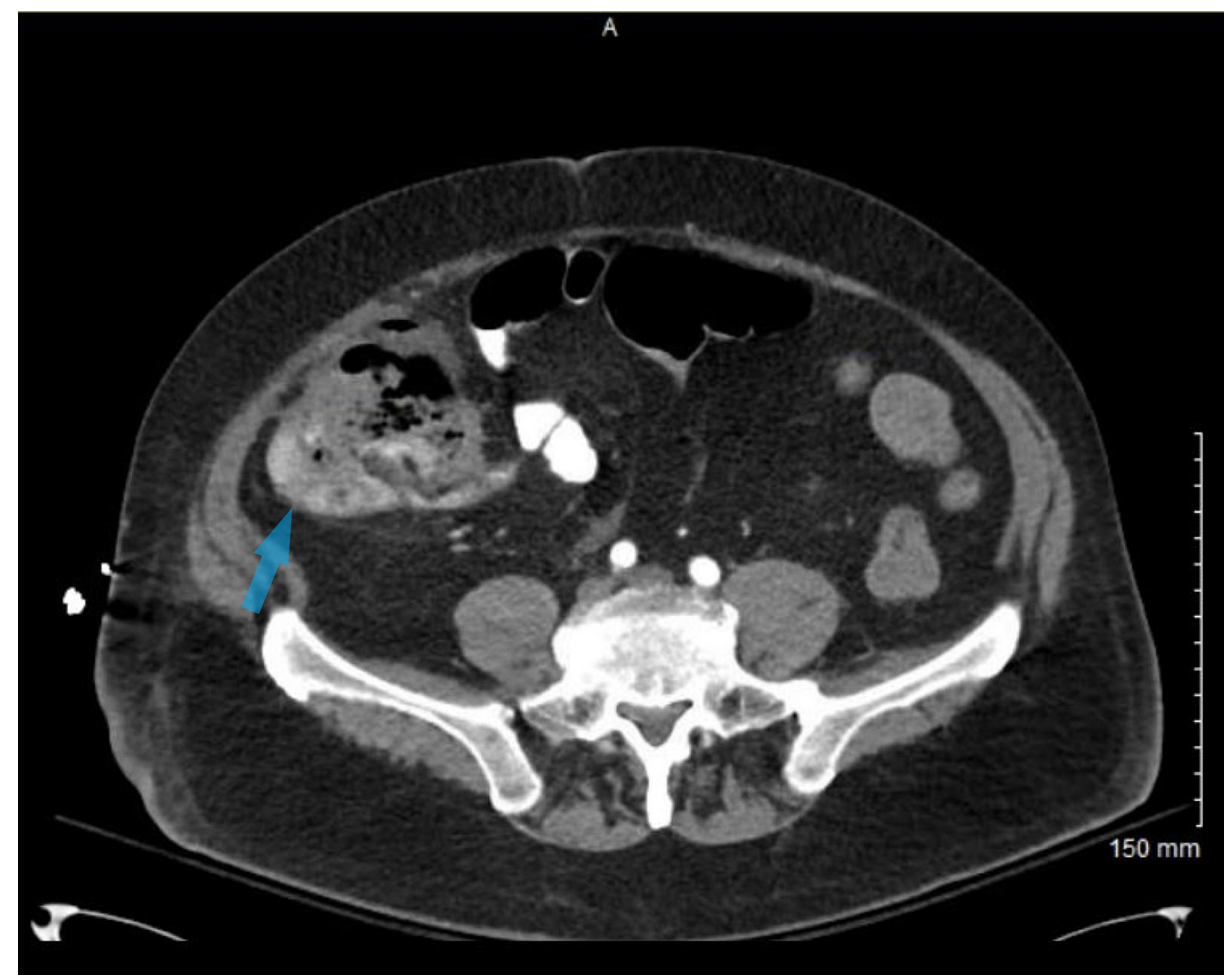

FIGURE 3: Axial view of a CT of the abdomen and pelvis with oral and intravenous contrast

An 8-cm cecal mass (Transparent blue arrow) with loss of the fat plane between the cecal mass and the anterior lateral right abdominal wall with possible invasion of the wall.

Colonoscopy exhibited multiple ulcers in the cecum and at the ileocecal valve (Figure 4).

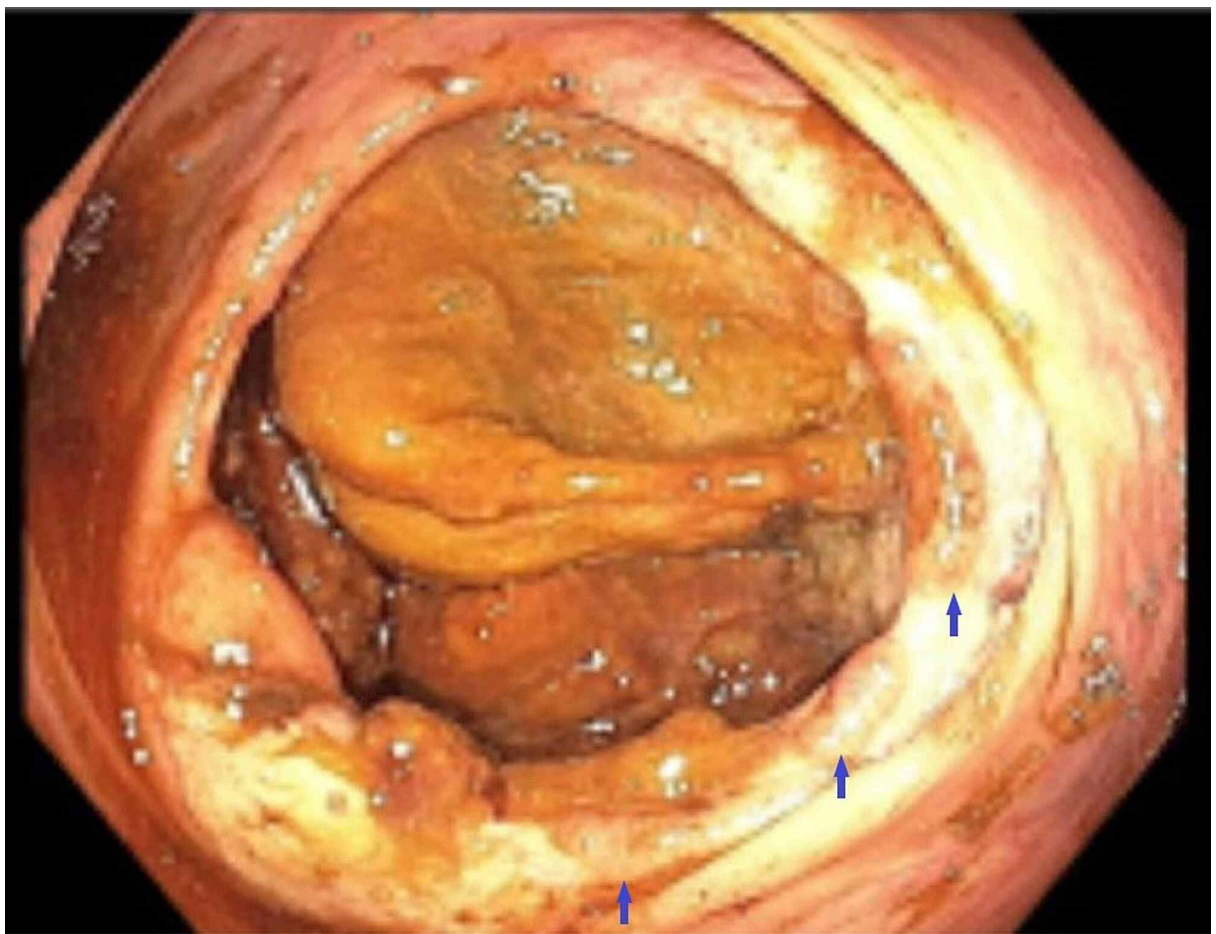

FIGURE 4: A colonoscopy image, at the level of the cecum

Multiple cecal ulcers (Blue arrows). 
Biopsies demonstrated focally ulcerated colonic mucosa with fibrinopurulent exudate highly suspicious for ischemic colitis. The patient improved on intravenous fluids and antibiotics. Repeat colonoscopy eight months later showed complete resolution of the colitis.

\section{Discussion}

IC is a nonocclusive disease resulting from a low flow state in the microvasculature of the colon. It represents the most common form of gastrointestinal ischemia, accounting for 50 to $60 \%$ of all ischemic events [1].

Typical patients with IC are not severely ill and present with vague symptoms (abdominal pain, the urge to defecate, maroon or bright red blood per rectum) that could be easily mistaken for infectious colitis or inflammatory bowel disease. The most common nonvascular CT findings in IC are segmental colonic wall thickening and pericolonic fat stranding [4]; these remain rather nonspecific signs [5]. Thus, early colonoscopy under minimal insufflation with biopsy remains the test of choice; findings can range from edema and erythema to pseudotumor or even gangrene. Serology (hemoglobin, WBC, BUN, LDH and sodium) has no diagnostic role and is only useful for risk stratification [6]. Overall, IC is a self-limited disease, as most cases resolve spontaneously and do not require specific therapy.

Over the last two decades, IRCI has been identified as a separate subgroup. IRCI arouses many questions most of which remain unanswered. The 'watershed areas' in the left colon have been historically thought to be the sites of poor perfusion making the right colon an unexpected area of primary ischemia. It has also been noticed that patients with IRCI usually carry a higher rate of comorbidities including CAD, atrial fibrillation and dialysis dependency [7]. Clinically, these patients present with a predominance of pain over rectal bleeding which is consistent with our two cases. In fact, the American College of Gastroenterology (ACG) guidelines strongly recommend considering the diagnosis of non-IRCI in patients with hematochezia. Compared with all other colon segments ischemia, IRCI has worse outcomes, a higher need for surgery, and a greater 30-day mortality rate [2,3]. Due to these specificities, IRCI pathophysiology has been hypothesized to be different from other IC. It is thought that IRCI could be secondary to a large vessel disease and could, in fact, herald, acute mesenteric ischemia. Longstreth and Hye [3] found in their retrospective study including 49 patients with IRCI that $11.4 \%$ of them were suffering from symptomatic large visceral artery occlusion causing abdominal angina. Cardiac arrhythmia and malignancies were also frequently diagnosed during the follow-up of these patients. Therefore, CT angiography is strongly recommended in patients with IRCI and, if not conclusive, a traditional splanchnic angiography should be considered. Note that CT with IV contrast is not appropriately timed for arterial visualization and is not sufficient in this setting.

As per ACG classification for IC, the presence of IRCI on imaging or colonoscopy represents alone a severe disease regardless of clinical presentation and serology: patients should be transferred to an intensive care unit, initiated on supportive treatment and broad-spectrum antibiotics and an emergent surgical consultation must be ordered [6].

IRCI is also famous for being the area of predilection for a rare variant called 'mass-forming' ischemic colitis (MFIC) by Khor et al. [8] and present in both of our patients. This IC mimics a tumor on CT or colonoscopy. It is thus crucial to have a high index of suspicion in recognizing this self-limited entity to prevent unnecessary surgery. According to some authors, this pseudotumor appearance could be due to submucosal edema and hemorrhage $[1,9]$. Colonoscopy with biopsy is the key to diagnosis. The management is the same for IRCI and complete resolution of the mass is expected within weeks.

\section{Conclusions}

IRCI is a rare clinical finding with a poorly understood pathophysiology. Nevertheless, it is accepted today that IRCI is the disease of vasculopath patients. The MFIC belongs almost exclusively to the IRCI and remains the subject of multiple controversies. In the clinical setting, the provider should be aware of this rare presentation of IC and of its unfavorable outcome. No specific treatment is available for the management of IRCI and MFIC, and supportive measures in an intensive care unit remain the rule.

\section{Additional Information \\ Disclosures}

Human subjects: Consent was obtained or waived by all participants in this study. United Health Services Institutional Review Board issued approval N/A. Dear Turki, We have reviewed this request and find it acceptable. Ronald Harris, DO. Chairman, IRB. Conflicts of interest: In compliance with the ICMJE uniform disclosure form, all authors declare the following: Payment/services info: All authors have declared that no financial support was received from any organization for the submitted work. Financial relationships: All authors have declared that they have no financial relationships at present or within the previous three years with any organizations that might have an interest in the submitted work. Other relationships: All authors have declared that there are no other relationships or activities that could appear to have influenced the 


\section{Cureus}

submitted work.

\section{References}

1. Zou X, Cao J, Yao Y, Liu W, Chen L: Endoscopic findings and clinicopathologic characteristics of ischemic colitis: a report of 85 cases. Dig Dis Sci. 2009, 54:2009-2015. 10.1007/s10620-008-0579-1

2. Sotiriadis J, Brandt LJ, Behin DS, Southern WN: Ischemic colitis has a worse prognosis when isolated to the right side of the colon. Am J Gastroenterol. 2007, 102:2247-2252. 10.1111/j.1572-0241.2007.01341.x

3. Longstreth GF, Hye RJ: Right-side colon ischemia: clinical features, large visceral artery occlusion, and long-term follow-up. Perm J. 2015, 19:11-16. 10.7812/TPP/15-024

4. Sherid M, Samo S, Sulaiman S, Husein H, Sethuraman SN, Vainder JA: Is CT angiogram of the abdominal vessels needed following the diagnosis of ischemic colitis? A multicenter community study. ISRN Gastroenterol. 2014, 2014:756926. 10.1155/2014/756926

5. Wolff JH, Rubin A, Potter JD, Lattimore W, Resnick MB, Murphy BL, Moss SF: Clinical significance of colonoscopic findings associated with colonic thickening on computed tomography: is colonoscopy warranted when thickening is detected?. J Clin Gastroenterol. 2008, 42:472-475. 10.1097/MCG.0b013e31804c7065

6. Brandt LJ, Feuerstadt P, Longstreth GF, Boley SI: ACG clinical guideline: epidemiology, risk factors, patterns of presentation, diagnosis, and management of colon ischemia (CI). Am J Gastroenterol. 2015, 110:18-44. 10.1038/ajg.2014.395

7. Brandt LJ, Feuerstadt P, Blaszka MC: Anatomic patterns, patient characteristics, and clinical outcomes in ischemic colitis: a study of 313 cases supported by histology. Am J Gastroenterol. 2010, 105:2245-2252. 10.1038/ajg.2010.217

8. Khor TS, Lauwers GY, Odze RD, Srivastava A: “Mass-forming” variant of ischemic colitis is a distinct entity with predilection for the proximal colon. Am J Surg Pathol. 2015, 39:1275-1281. 10.1097/PAS.0000000000000438

9. Brandt LJ, Katz HJ, Wolf EL, Mitsudo S, Boley SJ: Simulation of colonic carcinoma by ischemia . Gastroenterology. 1985, 88:1137-1142. 10.1016/s0016-5085(85)80072-X 